\title{
Beneficial effects of fasting on white adipose tissue inflammation and metabolic syndrome in obese subjects: review
}

\begin{abstract}
Obesity, a worldwide public health problem is often associated with the metabolic syndrome. Indeed, infiltration of immune cells within obese adipose tissues participates to the establishment of a chronic sub-inflammatory environment leading to insulin resistance. As in various inflammatory disorders, fasting regimens have demonstrated their beneficial effects in reducing obesity and its subsequent metabolic syndrome, through reduction of inflammation in white adipose tissues. In this review, we will highlight how caloric restriction can be protective against obesity associated inflammation and metabolic syndrome.

Background: Obesity is dependent on genetic factors, lifestyle, and diet. Increased incidence of obesity and its subsequent complications, such as type 2 diabetes (T2D), are a worldwide public health problem, as one third of the population in some developed countries is obese, i.e: having a body mass index (BMI) more than $30 \mathrm{~kg} / \mathrm{m}^{2}$. Interconnection of obesity with metabolic alterations or diseases, such as T2D, dyslipidemia, hypertension, and atherosclerosis is widely recognized, as the metabolic syndrome. ${ }^{1}$ Moreover, obesity is also known to be a risk factor of systemic or organ inflammation.
\end{abstract}

Keywords: obesity, white adipose tissue inflammation, caloric restriction, fasting, 17 cells, IL-17a, metabolic syndrome
Volume 4 Issue 6 - 2017

\author{
Marwa Chehimi,' Assia Eljaafari',2 \\ 'Univ-Lyon, CarMeN laboratory, , Université Claude Bernard \\ Lyon I, France \\ ${ }^{2}$ Clinical Research Department, Hospices civils de lyon, Centre \\ Hospitalier Lyon-Sud, France
}

\author{
Correspondence: Assia Eljaafari, Inserm U1060-CarMeN, 165 \\ chemin du grand Revoyet, 69310 Pierre Bénite, France, \\ Email assia.eljaafari@univ-lyon I.fr
}

Received: April 14,2017| Published: une 12, 2017
Abbreviations: TD, type 2 diabetes; BMI, body mass index; WAT, white adipose tissue; SVF, stromal vascular fractions; TLR-4, toll-like-receptor 4; MI, myocardial infarction

\section{Introduction}

White Adipose Tissue (WAT) inflammation is considered as the causal event leading to insulin resistance, and T2D. ${ }^{2}$ Indeed, obese WAT becomes heavily infiltrated by a variety of immune cells, ${ }^{3}$ with macrophage infiltration occurring at an advanced stage and driving inflammation. In contrast, $\mathrm{T}$ cells are likely to play a regulatory role ${ }^{5}$ since lean AT are infiltrated by anti-inflammatory $\mathrm{CD}^{4+} \mathrm{Foxp}^{3-}$ regulatory $\mathrm{T}$ cells (Tregs) and Th2 cells, secreting both IL-10, a cytokine known to improve adipocyte insulin sensitivity. ${ }^{6}$ In contrast, $\mathrm{CD}^{8+} \mathrm{T}$ cell accumulation in AT from obese subjects is known to precede macrophage infiltration, and contribute to WAT inflammation through macrophage recruitment. ${ }^{7}$ Besides their function as fat repositories, adipocytes can also be considered as endocrine cells that secrete numerous adipokines involved in the regulation of metabolic homeostasis, with IL-6 and leptin being pro-inflammatory while adiponectin is anti-inflammatory. ${ }^{8}$ Therefore, a crosstalk between adipocytes and immune cells that infiltrate WAT may govern homeostasis under physiological conditions, but contribute to the establishment of a chronic sub-clinical inflammation, a prerequisite for insulin resistance, during obesity. A recently discovered T-cell lineage playing a central role in WAT inflammation corresponds to the T-helper 17 (Th17) cell subset, ${ }^{9}$ which mainly secretes IL-17A and IL-17F pro-inflammatory cytokines. ${ }^{10} \mathrm{IL}-17 \mathrm{~A} / \mathrm{F}$ play a crucial role against extracellular bacterial or fungi infections but also induce secretion of other pro-inflammatory cytokines such as IL-1 $\beta$ and IL-6, by a variety of immune and non-immune cells, including monocytes, stromal cells, adipocytes and stem cells, which all express IL-17A/F receptors. ${ }^{11}$ Th17 cells have been implicated in the pathogenesis of various autoimmune diseases, such as psoriasis, multiple sclerosis and/or rheumatoid arthritis. ${ }^{12,13}$ Interestingly, IL-17A secretion was demonstrated to be increased not only in the periphery of obese patients, ${ }^{14}$ but also within their WAT. ${ }^{15}$ IL-17A has also been suggested to favor the development of insulin resistance, through downregulation of multiple pro-adipogenic transcription factor expression, such as PPAR $\gamma, \mathrm{C} / \mathrm{EBP} \alpha$, leading to inhibition of adipogenesis. ${ }^{16}$ Recently, we have reported that adipose stem cells (ASC) from obese, but not lean donors, contribute to Th17 promotion through contactdependent interactions and soluble factor secretion. ${ }^{17}$ ASC-mediated IL-17A secretion was associated with increased secretion of IL-1 $\beta$ by monocytes and IL-6 by ASC (Figure 1A). Interestingly, IL-17A secretion was also found to be increased in the stromal vascular fractions (SVF) of obese, but not lean subjects..$^{17,18}$

\section{Dietary restrictions and inflammation}

\section{Systemic effects}

Caloric restriction (CR) is an effective intervention in the management of inflammatory, and or auto-immune diseases. Indeed, fasting appears to play a role in reducing inflammation, likely resulting from different mechanisms. The possible antioxidative effect of fasting was evaluated by Faris, in 50 Ramadan fasters avoiding food for almost 12 hours per day during a 28 -day period. ${ }^{19}$ Systemic proinflammatory cytokines IL- $1 \beta$, IL- 6 , and TNF- $\alpha$, were measured 7-d, 21-d, and 1-month after Ramadan fasting. Reduced IL-1 $\beta$, IL-6, and 
TNF- $\alpha$ levels were observed after 21 day fasting and decreased IL- 6 levels were maintained 1 month after Ramadan fasting, indicating sustained effects of fasting on systemic inflammation. Supporting these results, another study by Dogan et al. demonstrated, that intermittent $\mathrm{CR}$ in rodents resulted in decreasing serum IL-6 and TNF- $\alpha$ levels by 24 -fold, and 11 -fold lower, respectively, as compared to ad-libitum fed mice, together with reducing IGF-I and leptin levels. ${ }^{20}$ Moreover, a positive correlation between IL- 6 /TNF- $\alpha$ levels and body weights was observed, suggesting that beneficial effects of CR may be related to regulation of pro-inflammatory cytokine pathways. Heat-generated advanced glycation end products (AGE) can also contribute to induction of inflammation in multiple organs, such as pancreas, heart, vessels, kidney and/or retina. They can be induced by dietary glycotoxins, but diet restriction was demonstrated to suppress their induction..$^{21}$ The reduction of inflammatory markers under CR might reduce metabolic syndrome linked to obesity and overfeeding. Finally, besides its effects on chemotherapy toxicity ${ }^{22}$ by reducing IGF-I, insulin, glucose levels, and increasing ketone body levels - fasting appears to protect against oxidative stress-induced DNA damage and carcinogenesis. ${ }^{23}$
A

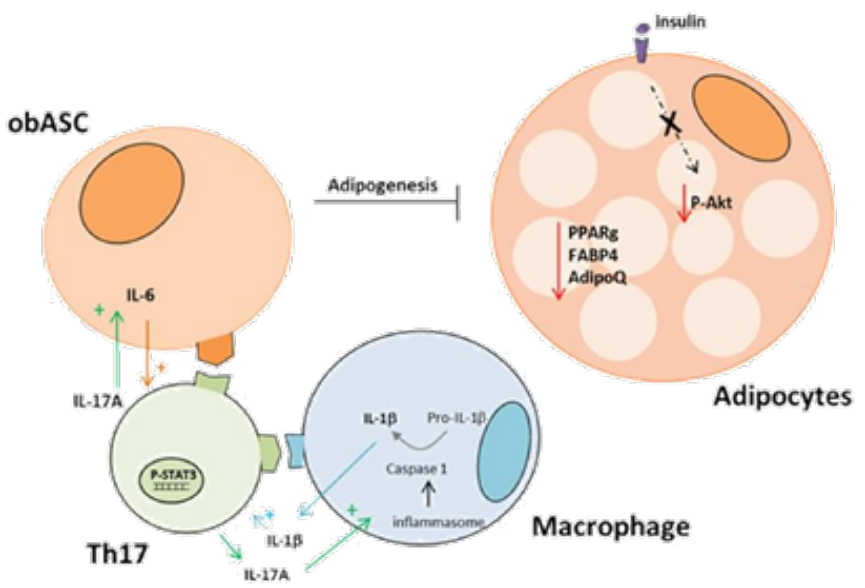

B

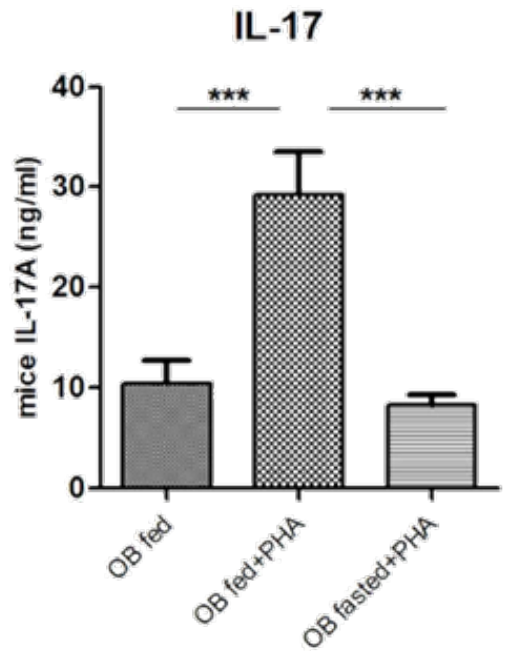

Figure I T helper cell (Th)I7 activation in obese adipose tissue (AT) is reversed by short-term fasting. (A) Obese adipose stem cells (ASC) polarize ATinfiltrating CD4+ T cells towards IL-I7A-secreting Th I 7 cells and increase IL- I $\beta$ secretion by monocytes, which amplify IL- I7A secretion. In turn, IL-6 secretion is increased in ASC, together with increased resistance to insulin, and inhibition of adipogenesis (from Eljaafari et al model). (B) ob/ob mice were fed or fasted overnight and IL-I7A secretion was evaluated in stromal vascular fraction derived from visceral AT after phytohemagglutin (PHA) activation during 48 hours. Short-term fasting mice markedly suppressed pro-inflammatory IL-I7A secretion.

\section{Effects of fasting on organ inflammation}

Obesity leading to metabolic syndrome is a risk factor for several diseases as liver, heart diseases, rheumatoid arthritis (RA) and stroke. In liver, alternate day food restrictions were shown to suppress the expression levels of Toll-like-receptor 4 (TLR-4) and nuclear factor $\kappa \mathrm{B}(\mathrm{NF}-\kappa \mathrm{B})$ protein in the liver and pro-inflammatory genes, such as IL- $1 \beta$, TNF- $\alpha$, and serum amyloid A (SAA). ${ }^{24}$

Similar results were obtained in heart, using an animal model of myocardial infarction (MI), where intermittent fasting was tested. The size of the ischemic infarct 24-h following permanent ligation of a coronary artery was significantly smaller and markers of inflammation, i.e. infiltration of leukocytes in the area at risk and plasmatic IL-6 levels, were reduced in intermittent fasted rats compared to controls. ${ }^{25}$ Moreover, intermittent fasting resulted in increased levels of circulating adiponectin after MI, with adiponectin being considered as an anti-inflammatory adipokine.

RA is a chronic inflammatory disease where synovium inflammation plays a predominant role. Obesity leading to metabolic syndrome is a risk factor of RA, since obese RA patients displayed more active and severe RA disease than lean RA patients. ${ }^{26,27}$ In RA patients, synovial levels of IL-6 are high. IL-6 has a preponderant role in regulating the acute phase response, activation of T-cells, and proliferation of synovial fibroblasts. A 7-day fasting in 10 patients with RA, significantly reduced serum IL- 6 and CRP levels, resulting in significant decrease of TNF- $\alpha$ in synoviocytes. ${ }^{28}$ In another study, 8-day fasting was clinically evaluated in RA patients and resulted in decreased disease activity scores. ${ }^{29}$

In stroke, pro-inflammatory cytokines and chemokines, are quickly produced by neuronal, glial and endothelial cells following an ischemic event, due to the presence of high levels of intracellular $\mathrm{Ca}^{2+}$ and ROS, in addition to a low oxygen environment in the brain (hypoxia). CR was shown to protect and improve neuronal survival from glutamate toxicity in rodent models through increasing neurotrophic factors such as BDNF and $\beta$-FGF resulting in the activation of transcription factor cyclic AMP response element binding (CREB) protein. ${ }^{30}$

\section{Effects of fasting on adipose tissue inflammation and metabolic syndrome}

AT responds dynamically to changes in nutrient availability, through triglyceride stockage or lipolysis. Thus overfeeding reduces lipolysis, and dyslipidemic subjects have larger subcutaneous adipocytes than controls. ${ }^{31}$ Food restriction appears able to (i) increase lipolysis resulting in reduction of adipocyte size, (ii) increase adiponectin secretion, and (iii) reduce leptin, IL- $1 \beta$, IL- 6 , VEGF- $\alpha$, MCP- 1 and CD-68 expression in WAT. ${ }^{32}$ Accordingly, insulin sensitivity was 
improved, together with decreased blood glucose levels, in a 6 months twice-weekly CR diet. ${ }^{33}$ IL-17A secretion is also increased in obese WAT, as reported by us and others..$^{10,14,15,17,18}$ Therefore, using our previously experimental model able to promote Th17 cell activation in obese WAT, ${ }^{17}$ we analyzed the effects of overnight fasting in genetically modified ob/ob mice and observed a marked reduction in IL-17A secretion (Figure 1B), suggesting that short-term fasting can be sufficient enough to inhibit Th17 cell activation in WAT. Finally, CR seems also able to reduce WAT inflammation even in non-obese individuals, as assessed by sterile inflammation models, where the effects of fasting on WAT inflammation have been tested in stressed rat. Indeed, fasting reduced visceral WAT TNF- $\alpha$, and subcutaneous WAT IL-1 $\beta$, as well as plasmatic insulin and leptin levels. ${ }^{34}$

Altogether these studies indicate that dietary restrictions may be useful for reducing the risk of acute or chronic inflammatory diseases, notably in obese individuals. Indeed, human and animal models as well, have enabled the demonstration that fasting regimens can modulate systemic, and/or tissue inflammatory processes, and improve metabolic markers.

\section{Acknowledgements}

None.

\section{Conflicts of interest}

The author declares there is no conflict of interest.

\section{References}

1. Despres JP, Lemieux I. Abdominal obesity and metabolic syndrome. Nature. 2006;444(7121):881-887.

2. Donath MY, Shoelson SE. Type 2 diabetes as an inflammatory disease. Nat rev Immunol. 2011;444(7121):881-887.

3. Osborn O, Olefsky JM. The cellular and signaling networks linking the immune system and metabolism in disease. Nature medicine. 2012;18(3):363-374.

4. Weisberg SP, McCann D, Desai M, et al. Obesity is associated with macrophage accumulation in adipose tissue. $J$ Clin Invest. 2003;112(12):1796-808.

5. Lumeng CN, Maillard I, Saltiel AR. T-ing up inflammation in fat. Nat med. 2009;15(8):846-847.

6. Feuerer M, Herrero L, Cipolletta D, et al. Lean, but not obese, fat is enriched for a unique population of regulatory $\mathrm{T}$ cells that affect metabolic parameters. Nat med. 2009;15(8):930-939.

7. Nishimura S, Manabe I, Nagasaki M, et al. CD8+ effector T cells contribute to macrophage recruitment and adipose tissue inflammation in obesity. Nat med. 2009;15(8):914-920.

8. Ouchi N, Kihara S, Funahashi T, et al. Obesity, adiponectin and vascular inflammatory disease. Curr Opin lipidol. 2003;14(6):561-566.

9. Fouser LA, Wright JF, Dunussi-Joannopoulos K, et al. Th17 cytokines and their emerging roles in inflammation and autoimmunity. Immunol rev. 2018;226:87-102.

10. Ahmed M, Gaffen SL. IL-17 in obesity and adipogenesis. Cytokine Growth Factor Rev. 2010;21(6):449-453.

11. Gaffen SL. Recent advances in the IL-17 cytokine family. Curr Opin Immunol. 2011;23(5):613-619.

12. Ouyang W, Kolls JK, Zheng Y. The biological functions of T helper 17 cell effector cytokines in inflammation. Immunity. 2008;28(4):454-467.

13. Eljaafari A, Miossec P. Cellular sides of acquired immunity (T cells). Chapter in Oxford Textbook of Rheumatology ( $4^{\text {th }}$ end), Oxford University Press, 2003; USA.
14. Sumarac-Dumanovic M, Stevanovic D, Ljubic A, et al. Increased activity of interleukin-23/interleukin-17 proinflammatory axis in obese women. Int J Obes (Lond). 2009;33(1):151-156.

15. Fabbrini E, Cella M, McCartney SA, et al. Association between specific adipose tissue CD4+ T-cell populations and insulin resistance in obese individuals. Gastroenterology. 2013;145(2):366-374.e1-e3.

16. Ahmed M, Gaffen SL. IL-17 inhibits adipogenesis in part via $\mathrm{C} / \mathrm{EBP} \alpha$, PPAR $\gamma$ and Krüppel-like factors. Cytokine. 2013;61(3):898-905.

17. Eljaafari A, Robert M, Chehimi M, et al. Adipose Tissue-Derived Stem Cells From Obese Subjects Contribute to Inflammation and Reduced Insulin Response in Adipocytes Through Differential Regulation of the Th1/Th17 Balance and Monocyte Activation. Diabetes. 2015;64(7):2477-2488.

18. Chehimi M, Robert M, Bechwaty ME, et al. Adipocytes, like their progenitors, contribute to inflammation of adipose tissues through promotion of Th-17 cells and activation of monocytes, in obese subjects. Adipocyte. 2016;5(3):275-282.

19. Faris MA, Kacimi S, Al-Kurd RA, et al. Intermittent fasting during Ramadan attenuates proinflammatory cytokines and immune cells in healthy subjects. Nutr Res. 2012;32(12):947-955.

20. Dogan S, Ray A, Cleary MP. The influence of different calorie restriction protocols on serum pro-inflammatory cytokines, adipokines and IGF-I levels in female C57BL6 mice: short term and long term diet effects. Meta Gene. 2017;12:22-32.

21. Vlassara H, Cai W, Crandall J, et al. Inflammatory mediators are induced by dietary glycotoxins, a major risk factor for diabetic angiopathy. Proc Natl Acad Sci U S A. 2002;99(24):15596-15601.

22. Raffaghello L, Lee C, Safdie FM, et al. Starvation-dependent differential stress resistance protects normal but not cancer cells against high-dose chemotherapy. Proc Natl Acad Sci U S A. 2008;105(24):8215-8220.

23. Longo VD, Mattson MP. Fasting: molecular mechanisms and clinical applications. Cell Metab. 2014;19(2):181-192.

24. Yang W, Cao M, Mao X, et al. Alternate-day fasting protects the livers of mice against high-fat diet-induced inflammation associated with the suppression of Toll-like receptor 4/nuclear factor $\kappa \mathrm{B}$ signaling. Nutr Res. 2016;36(6):586-593

25. Wan R, Ahmet I, Brown M, et al. Cardioprotective effect of intermittent fasting is associated with an elevation of adiponectin levels in rats. $J$ Nutr Biochem. 2010;21(5):413-417.

26. Stavropoulos-Kalinoglou A, Metsios GS, Koutedakis Y, et al. Obesity in rheumatoid arthritis. Rheumatology (Oxford). 2011;50(3):450-462.

27. Jhun JY, Yoon BY, Park MK, et al. Obesity aggravates the joint inflammation in a collagen-induced arthritis model through deviation to th17 differentiation. Exp Mol Med. 2012;44(7):424-431.

28. Fraser DA, Thoen J, Djøseland O, et al. Serum levels of interleukin-6 and dehydroepiandrosterone sulphate in response to either fasting or a ketogenic diet in rheumatoid arthritis patients. Clin Exp Rheumatol. 2000;18(3):357-262.

29. Michalsen A, Riegert M, Ludtke R, et al. Mediterranean diet or extended fasting's influence on changing the intestinal microflora, immunoglobulin A secretion and clinical outcome in patients with rheumatoid arthritis and fibromyalgia: an observational study. BMC Complement Altern Med. $2005 ; 5: 22$.

30. Fann DY, Ng GY, Poh L, et al. Positive effects of intermittent fasting in ischemic stroke. Exp Gerontol. 2017;(89):93-102.

31. Varady KA, Roohk DJ, Loe YC, et al. Effects of modified alternate-day fasting regimens on adipocyte size, triglyceride metabolism, and plasma adiponectin levels in mice. $J$ Lipid Res. 2007;48(10):2212-2219.

32. Romero Mdel M, Fernández-López JA, Esteve M, et al. Different modulation by dietary restriction of adipokine expression in white adipose tissue sites in the rat. Cardiovasc Diabetol. 2009;8:42. 
33. Harvie MN, Pegington M, Mattson MP, et al. The effects of intermittent or continuous energy restriction on weight loss and metabolic disease risk markers: a randomized trial in young overweight women. Int J Obes (Lond). 2011;35(5):714-727.
34. Speaker KJ, Paton MM, Cox SS, et al. A Single Bout of Fasting (24h) Reduces Basal Cytokine Expression and Minimally Impacts the Sterile Inflammatory Response in the White Adipose Tissue of Normal Weight F344 Rats. Mediators of Inflammation. 2016(2016):13. 